\title{
STAR WEEK: A Successful Campaign in Japan
}

\author{
Junichi Watanabe \\ National Astronomical Observatory of Japan, Osawa, Mitaka, \\ Tokyo 181-8588, Japan \\ email: jun.watanabe@nao.ac.jp
}

\begin{abstract}
A campaign of the Star Week, set as August 1 - 7, has been started in 1995 throughout various life-learning facilities. Astronomical facilities including planetariums, science museums, and public observatories has been participating in this campaign, together with amateur astronomers clubs or private observatories. More than 300 astronomical events such as star watching parties were coordinated for general public every year. The Star Week has been grown up to one of the most successful campaigns not only for for astronomy but also for basic sciences in Japan over this 15 years. In this paper, a brief history and the present status of the Star Week are introduced together with the with some statistics.
\end{abstract}

Keywords. Public Outreach, Campaign, Education

\section{Introduction}

The circumstance of Japanese astronomical education is quite unique such that there are several hundreds of astronomical facilities for general public, including planetariums, museums, and public observatories. Especially the number of the public astronomical observatories increased rapidly in 1990s (Kuroda 1993). While the number of active planetarium is about 300(White Paper on Planetariums 2005, http://www . planetarium.jp/ pub/wbook2005/index.html) that of public observatories is more than 90 excluding science museum (White Paper on Public Astronomical Observatories 2006). These facilities are basically operated by local governments of prefectures, cities, towns, or villages. This situation means that there is no formal connection among the facilities. Actually, the planetariums had three societies depending on the planetarium companies. One society is there for science museums, which is exclusively for museum. For public observatories a Japan Public Observatories Society has been established in 2005, and before that they have only annual meeting once a year for communicating each other. Hence, there is no widely-covered society or no action to connect all these facilities effectively until 1995 .

In order to do so, and to make good collaborations especially for astronomical education purpose, the Star Week campaign was originally proposed by the author in June 1995 at the annual meeting for public observatories (Watanabe et al. 1995). Many attendee agreed with this new idea to set the week for star watching or related activities all over Japan, collaborating with different kinds of facilities or amateur astronomers' clubs. The steering committee was made just after the meeting, and started activities from the summer of 1995. Since then, the Star Week becomes a unique opportunity for connecting all kinds of astronomical facilities together with astronomical amateurs involved, and has continued successfully over 15 years. This activity and the developed network became a basis of various activities performed during the International Astronomy Year 2009 in Japan. Here in this paper, this successful campaign is introduced together with some statistics. 


\section{Overview of the Star Week}

\subsection{History}

The idea of the Star Week was coming to the author's mind from the similar campaign "Bird Week", which was originally promoted as "Bird Day" similar to U.S. "Bird Day" (Babcock 1901) by an American researcher, Dr. O. L. Austin who came to Japan as a members of GHQ, in 1947. He suggested to make Japanese bird day for spreading correct knowledge and for protection of natural birds from expected development after the World War II. This might be regarded as a first environmental campaign in Japan. In 1950, the Bird Day was changed to the "Bird Week" to strengthen its campaign, which was set from May 10 through May 16 every year. Many events such as symposium, lectures, painting contest and bird watching are held during this week all over Japan.

Under the circumstances mentioned in the introduction, the author thought that similar campaign could be carried out by connecting various existing facilities over Japan. At the 4th annual meeting of public observatories was held in Gifu prefecture in June 12 - 14. The first half meeting was held at Gifu Astronomical Observatory, which has been founded basically for general public. In the meeting, the author was invited to be a panelist of the panel discussion on "role of public observatories in the society", where I introduced my idea (Watanabe et al. 1995). The steering committee was constructed just after the end of the meeting, and the secretariat office was organized in the Pubic Relations Office, which had been established in 1994, in the National Astronomical Observatory of Japan (hereafter, NAOJ, Ono et al., 2006). Actually the Public Relations Office had been established by the author in 1994 (Watanabe 1996)! in the NAOJ. Even if we started late in the first year of 1995, more than 100 facilities participated in this campaign, and about 200 astronomical events such as star parties were coordinated for general public. It was definitely a success at the initial phase of the Star Week.

The main task for steering committee of the Star Week was to call participating facilities or clubs and to collect information of the events related to astronomy education as much as possible. The information on many existing events performed independently had been collected and summarized as Star Week's events. The committee made press release of the obtained information to press or media, or directly to general public with using campaign web site maintained by the committee. A original poster and calender were printed and distributed to the participating facilities or groups, financially supported by the NAOJ. The original souvenirs such as post cards and T-shirts were also produced by members of steering committee. The author appeared in the TV program of the NHK dealing with the aspect and point under discussion in the society on August 5th, 1997, where he gave his opinion on the meaning of the Star Week.

Five years after the start of the Star Week, we had a chance to review the first-phase activities at the annual meeting of the public obervatories held at Saji Astronomical Observatory, Tottori in June $14-16,2000$. we had a special session III on the Star Week, in which we presented a present status (Namiki 2000), a statistical summary of the Star Week in 5 years (Ono 2000), and the future plan (Watanabe 2000). In this session there was an intense discussion on three aspects. One was the period of the Star Week in August 1-7, when it is usually expected that most part of Japan should be good weather after the rainy season together with the strong reason that this period located within the summer holiday of school all over Japan. The validity of this period had been intensively discussed from various perspective such as number of visitors for facilities, weather, and lunar phase. Although there was a strong opinion to change the period depending on the lunar phase in order to avoid the moon light, another insisted that the Moon itself should be watched as one of astronomical bodies. Finally we decided to fix the period 
for Star Week from August 1 through 7 mainly because many people can remember Star Week and easier to schedule any plan for various educational events. Other aspect discussed in this session was the method of registration for participation, which was a little complicated process at that time. We decided to try to simplify this process for calling wider participation. Especially in 2009, the registration for the individual events can be done through the IYA2009 web site, with cooperating with the IYA2009 planning committee where the author is the chairman. The third aspect was the participation from commercial companies, travel agencies, hotels or related to the economical business. However, no intense discussion occurred because originally only a few examples were there on this topic. In order to strengthen the Star Week, we decided not to reject such partners or participations from commercial companies. Some hotels or Japanese style Inns which has telescopes participated in the Star Week since then. Also the campaign song "COSMOS" made by musical group, Aquamarine, was determined, and this song became one of the popular "Chorus tune" in the public school.

\subsection{Purpose}

There may be no need to recall the purpose of this Star Week campaign because it is similar to the purpose of the IYA 2009. However, we have special reason to do so because Japan is one of the worst countries for light pollution. Especially most of children have no experience of seeing Milky Way. Let them see the real stars. Let them feel the universe by inviting them to the related astronomical facilities located all over Japan. For realizing this purpose, the Star Week campaign helps educators to realize such dreams. Many facilities also hope to increase the number of visitors, by arranging various astronomical events in each facility. This is one of the motivation of the beginning of the Star Week. Such outreach program continuing after the IAY 2009 should give opportunity for general public to understand the excitement of the astronomy.

\section{Statistics}

The number of the participating facilities is increasing with time, and 230 facilities performed 520 events in 1999, and became 217 facilities with 540 events registered (Watanabe et al. 2003). Figure 1 shows the variation of the numbers of registered facilities or groups together with events. After the peak at around 2000, the number is almost constant. Figure 1 shows the time variation of the registered numbers to the Star Week. One of the reason of the decreasing numbers recently is mainly due to the change of the managing system of many public facilities, where the local governments are trying to cut the maintaining cost by asking to the commercial companies.

Figure 2 shows the ratio of different kinds of registered groups in the Star Week 2008. While the $64 \%$ is the public facilities which is operated by the local governments, amateur astronomers' clubs occupied up to $19 \%$ of total, which means that the Star Week campaign has been accepted not only to the educators or curators working in the public facilities, but also to the general amateurs. These people do frequently hosted the star watching parties or star festival all over Japan. Sometimes the number of visitors becomes more than ten thousand in certain star festivals. Unfortunately, we did not collect any information on visitors for the Star Week's events mainly due to the uncertainties of the numbers especially for the festival until now. The category "private facility includes the private public observatories operated voluntarily by amateur or commercial companies, hotels or Inn having telescopes, and religious facilities such like temples or shrines. The category "!others" include the branch observatories of the NAOJ and other facilities of government-related facilities. 


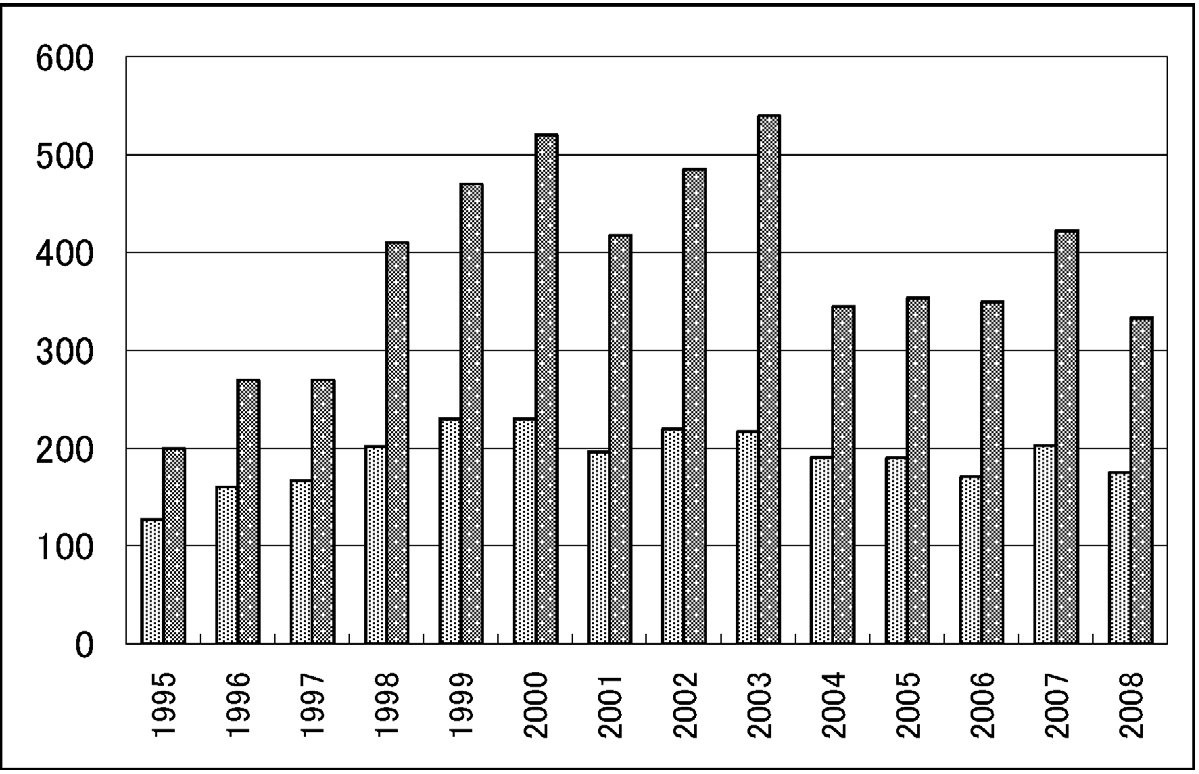

Figure 1. Total numbers of registered groups(thin color) and events(thick color) of the Star Week by each year.

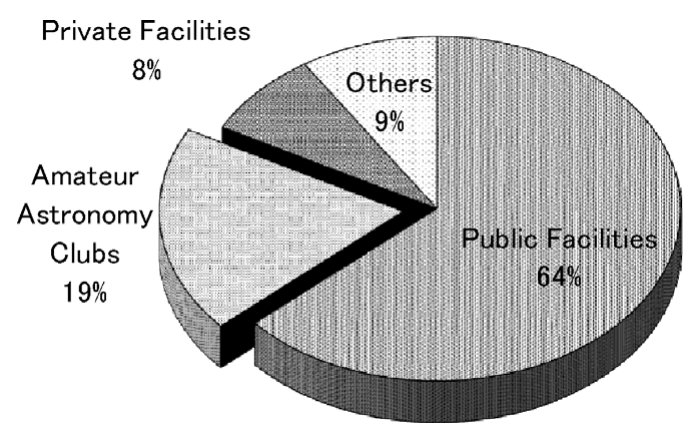

Figure 2. Type ratio of the registered groups or facilities for the Star Week in the case of 2008.

From viewing the such statistics, it is safely said that the Star Week has been grown up to one of the most successful campaigns not only for for astronomy but also for basic sciences in Japan.

\section{Future Scope}

The Star Week was the first trial in Japan to cooperate among lifelong-learning facilities related to the astronomy as one means of outreach activities by developing a original network. It hopefully provides opportunities for the general public to visit these astronomical facilities and watch the star-filled night skies during summer vacation through this campaign in cooperation with those facilities, general astronomy enthusiasts and their associations, which resulted in more than 400 events in average held during this period. It is safely said that this Star Week is a successful campaign until now, and as outreach activity in astronomy, and may play important role for further development of the similar activities in other filed. The high activity of the IYA2009 in Japan is partly based on the accumulated experiences in the star week. 
However, when we try to continue it, there are several problems. One of them is the lack of the fundings. The Public Relations Office of the NAOJ is playing important role for the Star Week by maintaining secretariat office along with some internal funds of the level about million yen per year. This fund is extremely small for extending such campaign to the higher level. Another problem is how to stimulate general people who are not originally interested in stars. Fortunately the IYA2009 can be a good turning point in this aspect. There are many excellent plans of outreach activities to rather noninterested people in Japan, and resulted in a great success. The Star Week committee tries to becomes a successor of the new wave kicked off by the IYA 2009. We try to solve these problems under a given circumstances, in order to let general public know the excitements of the universe, and beauty of the stars.

\section{Acknowledgements}

The author thanks especially Ms. T. Ono, Ms. N. Ishikawa, Mr. M. Sato and Mr. A. Koike, who helped organising the "Star Week" campaigns at the secretariat's office. He is also grateful to all the members of present steering committee: Mr. M. Asahi, Ms. N. Ono, Mr. O. Iiyama, Mr. K. Kawai, Mr. M. Furuya, Mr. Y. Matsumoto, Mr. J. Terazono, Mr. T. Yoshida, Mr. T. Yasuda, and Mr. A. Miyamoto. He gives special thanks to all the past members of the steering committee: Mr. M. Koishikawa, Mr. H. Oono, Ms. Y. Nozaki, Ms. M. Goto, Mr. H. Toda, Mr. M. Sakamoto, Dr. T. Horaguchi, Dr. K. Saijyo, Mr. K. Mishima, Mr. T. Arai, Mr. H. Gonoi, Mr. M. Yamanishi, Mr. T. Hamane, Dr. H. Obayashi, Dr. H. Agata, Mr. S. Ootomo, Mr. M. Ioroi, Dr. K. Ayani, Mr. T. Kiuchi, Mr. T. Kuroda, and H. Taguchi.

This paper is dedicated to Mr. Mitsuo Namiki, who unfortunately passed away in 2006, and who worked at the secretariat of "Star Week" from its very beginnings.

\section{References}

Babcock, C. A. 1901, Bird day; how to prepare for it (supplement of school) (Oil City, Pennsylvania: Silver Burdett and Company)

Kuroda, T. 1993, Ann. Rep. Nishi-Harima Astron. Obs., 3, 46

Namiki, M. 2000, in Proc. 9th annual meeting for Public Observatory (held at Saji Astronomical Observatory, June 14-16, 2000), p. 9 (in Japanese)

Ono, T. 2000, in Proc. 9th annual meeting for Public Observatory (held at Saji Astronomical Observatory, June 14-16, 2000), p. 10 (in Japanese)

Ono, T., Agata, H., Watanabe, J. \& Fukushima, T. 2006, in 25th ISTS (International Symposium on Space Technology and Science) (4-11 June 2006, Kanazawa, Japan), abstract ISTS 2006-u-08

Watanabe, J. 1996, Astronomical Herald (Astronomical Society of Japan), 89, 335 (in Japanese)

Watanabe, J. 2000, in Proc. 9th annual meeting for Public Observatory (held at Saji Astronomical Observatory, June 14-16, 2000), p. 11 (in Japanese)

Watanabe, J. 2006, in Innovation in Teaching/Learning Astronomy Methods, 26th General Assembly of the IAU, (17-18 August 2006, Prague, Czech Republic), SPS2, abstract No. 89

Watanabe, J. Yuasa, S., Fujita, Y. \& Funakoshi, H. 1995, in Proc. 4th annual meeting for Public Observatory (held at Gifu and Nishimino Astronomical Observatory, Jun 12-14 1995), p. 36 (in Japanese)

Watanabe, J., Ono, T. \& Star Week Committee 2003, in Effective Teaching and Learning of Astronomy, 25th General Assembly of the IAU (24-25 July 2003, Sydney, Australia), Special Session 4, abstract No. 2099, p. 59 